\title{
Psychophysiological Stress Assessment Among On-Duty Firefighters
}

\author{
Susana Rodrigues, Duarte Dias, Joana S. Paiva, and João P.S. Cunha, Senior Member, IEEE
}

\begin{abstract}
Firefighting is a hazardous profession commonly exposed to high stress that can interferewith firefighter's health and performance. Nevertheless, on-duty stress levels quantitative evaluations are very rare in the literature. In order to investigatefirefighters' ocaupational health in terms of stress perceptions, symptoms, and quantified physiological reactions under real-world conditions, an ambulatory assessment protocol was developed. Therefore, cardiac signal from firefighters $(N=6)$ was continuously monitored during two shifts within a working week with a medical clinically certified equipment (VitalJacket ${ }^{\circledR}$ ), allowing continuous electrocardiogram (ECG) and actigraphy measurement. Psychological data were collected with an android application, collecting potential stressful events, stress symptoms, and stress appraisal. A total of 130 hours of medical-quality ECG were collected, from which heart rate variability (HRV) metrics were extracted and analyzed. Statistical significant differences were found in some HRV metrics - AVNN, RMSSD, pNN50 and LF/HF - between events and non-events, showing higher levels of physiological stress during events $(p<0.05)$. Stress symptoms increase from the beginning to the end of the shift (from $1.54 \pm 0.52$ to $2.01 \pm 0.73$ ), however the mean stress self-perception of events was very low $(3.22 \pm 2.38$ in a scale ranging from 0 to 10). Negative and strong correlations were also found between stress symptoms and some time-domain ECG measures (AVNN, SDNN and pNN50). It can be concluded that stress may not always be detected when using merely self-reports. These results enhance the importance of combining both self-report and ambulatory high-quality physiological stress measures in occupational health settings. Future studies should investigate not only what causes stress but also its impact on health and well-being of these professionals, in order to contribute to the design of efficient stress-management interventions.
\end{abstract}

\section{INTRODUCTION}

Firefighters (FFs) are considered a high-risk occupational group, since they perform their typical occupational activities in very challenging, unpredictable emergency conditions, exposing them to severe stress [1]. Existing research has demonstrated that stressors faced by FFs have an impact on their physical and psychological welfare [2]. In fact, according to a recent report by the Federal Emergency Management Agency (2015), 67\% of the fatalities among FFs in 2014 could be attributed to stress and overexertion [3].

Stress can be defined as a process, whereas a situation is perceived as exceeding one's individual resources or endangers the person's well-being [4]. Stress perceptions are likely to activate physiological responses. Cannon was one of

*Research supported by national funds through the FCT Fundação para a Ciência e Tecnologia (Portuguese Foundation for Science and Technology) within the project VR2Market CMUP-ERI/FIA/0031/2013 and FCT PhD grant $\mathrm{PD} / \mathrm{BD} / 135023 / 2017$.

S. Rodrigues is with INESC Technology and Science, Porto, Portugal (email: scpr@inescporto.pt)

D. Dias is with INESC Technology and Science, Porto, Portugal (email duarte.f.dias@inesctec.pt) the first physiologists to explore the impact of stress, by describing the instinctual "fight-or-flight" response [5]. When a danger is perceived, the autonomic nervous system (ANS) is activated: the parasympathetic nervous system is inhibited and the sympathetic nervous system is triggered. This process leads to the excretion of stress-related hormones leading to vasoconstriction of blood vessels, increased blood pressure, increased muscle tension and heart rate (HR) and a decrease in heart rate variability (HRV). When the stressor is no longer present, a sympathovagal balance is reestablished through homeostasis between the parasympathetic and sympathetic system [6]. Among a widespread range of physiological indicators (e.g., blood pressure, cortisol, skin conductance), HRV has been proposed as a feasible and reliable measure to assess physiological stress responses [7]. HRV is related to the beat-to-beat changes in heart rates or heartbeat intervals, which has been recognized as an instantaneous quantitative measure of ANS activity associated with stress [8]. However, little agreement exists in ascertaining the best HRV classifiers for stress [7]. The operationalization and understanding of the stress process is somewhat complex. Hence, psychophysiological literature in this area proposed the combination of subjective measures of stress with objective and quantified physiological parameters $[9,10]$.

Based on previous recommendations, the current study developed a multimethod ambulatory stress approach for the assessment of FFs stress during their daily work duties. Taking this into account, the current method consists of a combination of self-reports with electrocardiogram (ECG) data analysis, relying on user-friendly and non-intrusive technology adapted to these professionals needs and requirements for the typically harsh environments they work. The current study aims to understand psychophysiological stress among on-duty FFs. Additionally, considering the lack of agreement in the literature on the choice of the best HRV parameters that mirrored stressful responses, the current study will also identify those parameters that change significantly under the influence of stress.

\section{MATERIALS AND METHODS}

\section{A. Acquisition system}

Six male FFs (mean age $29.50 \pm 8.55$ ) from a Portuguese Fire unit volunteered to participate in this study. The exclusion criteria for the study were participants having a history of cardiovascular disease and/or taking prescription drugs known to affect cardiovascular function. Participants underwent

J.S. Paiva is with INESC Technology and Science, Porto, Portugal (email: jipaiva@inesctec.pt).

J.P.S. Cunha (corresponding author) is with INESC Technology and Science, Porto, Portugal and Faculdade de Engenharia of Universidade do Porto, Portugal (e-mail: jcunha@ieee.org). 
ambulatory monitoring on 2 shifts of the same work week. They were equipped with a wearable t-shirt that is a wearable ECG monitor - VitalJacket ${ }^{\circledR}[11,12]$ - and an electronic diary based on a custom made Android smartphone application that enable data synchronization and event marking. Fig. 1 illustrates the monitoring system workflow.

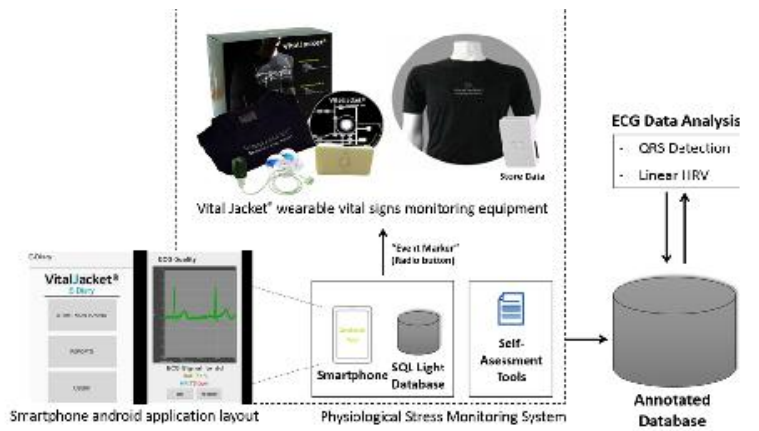

Fig. 1. System block diagram explaining the monitoring system functioning workflow.

This system pairs with Vital Jacket ${ }^{\circledR}$ via Bluetooth and enables the exact time annotation of events in the device, using "Radiobuttons". This action allows to register the events in the device and synchronize them with the ECG that is being acquired simultaneously. The android application stores all the information about the events in an SQL Light DataBase, from where a report of the event data can be generated and exported for processing and analysis, always warranting the synchronization between those events information and the data collected by the Vital Jacket ${ }^{\circledR}$.

\section{B. Self-reports}

Demographic and medical surveys were used to assess participants' current health state. The Android smartphone application includes a stress symptoms questionnaire [13]. This instrument includes four questions related to physical aspects and four questions related to cognitive aspects of stress (ranging from ' 1 - not felt at all' to ' 5 - extremely felt'). An example of a physical symptom question is 'I have a stiff neck'; an example of a cognitive symptom question is 'I lack concentration'. These questions were answered at the beginning and at the end of the shift, aiming to evaluate whether there were changes in stress symptoms experienced from the beginning to the end of it. Additionally, a Visual Analogue Scale (VAS) [14] was used after each event and before and after each shift to assess perceived stress levels on a 10 levels scale (ranging from ' 0 - None' to ' 10 - As bad as it could be'). Work stress was classified as high when VAS > 6 and low when VAS $<3$ [15]. The FFs also provided information regarding the experienced events in an openended event question displayed in the smartphone application.

\section{ECG data}

Physiological stress was quantified based on diverse measures of HRV. These measures are in accordance with the guidelines presented by the Task Force of the European Society of Cardiology and the North American Society of Pacing and Electrophysiology [16] that are viable for stress assessment: AVNN - Average of (normal-to-normal) NN intervals (expressed in milliseconds (ms)); SDNN - Standard Deviation of all NN intervals (ms); RMSSD - Root Mean
Square of Differences between successive NN intervals (ms); pNN50 - the percentage of pairs of adjacent normal sinus (NN) intervals differing by more than $50 \mathrm{~ms}$ and LF/HF Ratio - this is the ratio between the power of Low Frequency and High Frequency ECG components.

Decreased values of AVNN, SDNN, RMSSD, pNN50 and increased values of LF/HF are indicative of stress [7]. Table 1 sum up these HRV parameters and their trend under stress.

TABLE I. HRV measures and their trend under stress [7].

\begin{tabular}{|l|c|c|}
\hline Type & Measure & $\begin{array}{c}\text { Features trend } \\
\text { under stress }\end{array}$ \\
\hline Time-domain & AVNN & $\downarrow$ \\
& SDNN & $\downarrow$ \\
& RMSSD & $\downarrow$ \\
& pNN50 & $\downarrow$ \\
\hline Frequency-domain & LF/HF & $\uparrow$ \\
\hline
\end{tabular}

\section{DATA ANALYSIS}

\section{A. Statistical analysis}

Data were statistical analyzed using IBM SPSS AMOS (v.24) software. Taking into account the few number of population samples, some parameters failed in the normality test, so all parameters were analyzed using non-parametric statistical tests [17]. Therefore, Wilcoxon-Signed Test was performed to compare means between the variables. Spearman correlation was also used in order to find significant correlations between psychological variables and physiological data (ECG-related measures).

\section{B. ECG data analysis}

In order to extract heartbeat data from the ECG recordings, we used the Biodevices, S.A. ECG analyzer, which has an algorithm based on Pan and Tompkins approach [18] that detects each heartbeat in the ECG recording, locating the ' $R$ ' points of the ECG waveform. Using this analyzer, the RR intervals were extracted. A simple verification according to Clifford et al [19] was implemented to verify if all the RR intervals were physiologically valid. This procedure can eliminate any possible mistake made by the analyzer that could occur in case of a noisy ECG signal. The RR intervals that have physiological validation are named normal-to-normal (NN) intervals.

Data were collected during the entire shift, including nonevents and events. HRV metrics - AVNN, SDNN, RMSSD, pNN50, and LF/HF were computed using 5 minutes' windows, without overlapping and excluding participant's movement data. However, since the total number of 5-minute ECG blocks among all subjects was not balanced between non-events and events, a minimum number of 5-minute ECG blocks was chosen randomly from the raw samples distribution of HRV shift analysis. Hence, 10 blocks of 5minutes per person, for each condition (non-events $v s$ events) were considered in the statistical analysis. This allowed to perform statistical comparisons and correlations considering a balanced sample number per condition.

\section{RESULTS AND DISCUSSION}

A total of 130 hours of clinical-grade ECG was obtained during the fire summer season in Portugal (August to October, 
2017). A total of 12 fire events were reported by the FFs, which was expectable since they were part of a special team dedicated to fire combat.

Regarding the ECG analysis, results from WilcoxonSigned test revealed statistical significant differences for AVNN, RMSSD, pNN50 and LF/HF when comparing events and non-events $(\mathrm{p}<0.05)$ (Fig. 2). Values below shown in Fig. 1 are normalized to Voss's et al study $(\mathrm{N}=1906)$ for healthy population (controlling for age and gender) [20].

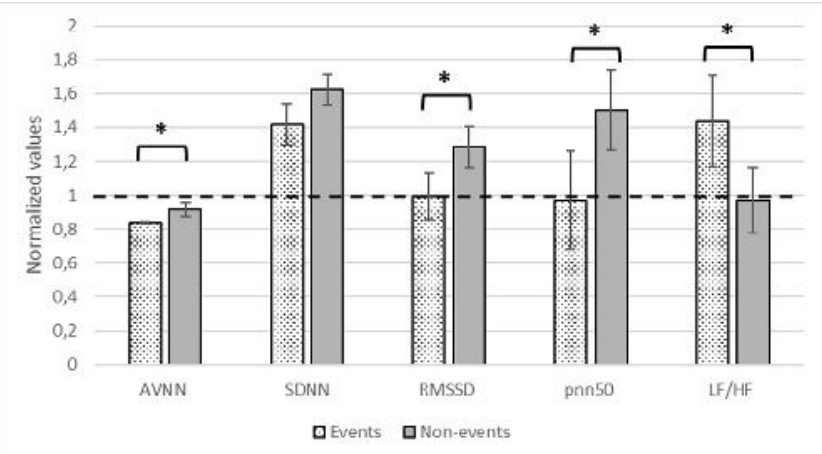

Fig. 2. Normalized mean values for ECG-derived measures using Voss 's et al. study with an healthy population. Values of AVNN, SDNN, RMSSD and pNN50 below dashed line are suggestive of stress. Values of LF/HF above the dashed line could also be suggestive of stress; *statistical significant differences using Wilcoxon-Signed test, $\mathrm{p}<0.05$.

The statistical significant lower values of time-domain measures during events (with the exception of SDNN), and higher values of $\mathrm{LF} / \mathrm{HF}$ during events, when comparing to non-events suggest that fire events cause physiological stress arousal [7]. However, the overall mean of stress self-perceived VAS after fire events was low $(M=3.22)$ (Table 2).

TABLE II. Self-reports mean values and standard deviation of selfreports

\begin{tabular}{|c|c|c|c|}
\hline & $\begin{array}{c}\text { Beginning of } \\
\text { the shift }\end{array}$ & $\begin{array}{c}\text { End of } \\
\text { shift }\end{array}$ & After event \\
\hline Stress VAS (0-10) & $1.69 \pm 1.91$ & $2.56 \pm 2.37$ & $3.22 \pm 2.38$ \\
\hline $\begin{array}{c}\text { Stress symptoms } \\
\text { questionnaire (1-5) }\end{array}$ & $1.54 \pm 0.52$ & $2.01 \pm 0.73$ & n.a \\
\hline
\end{tabular}

The possible mismatch between self-reports levels after events and their physiological responses, could be related with the fact that the reported events are regularly experienced by FFs, thus they do not perceive them as stressful, but rather as a routine part of their daily work. Similar results were reached in a study with police officers using a similar methodology [21].

Complementarily to stress perceptions and physiology, stress symptoms were also analyzed. Findings suggest that there was a slight increase in symptoms from the beginning to the end of the shift (Table II). However, this increase was not statistically significant, possibly due to the reduced sample size. The observed tendency supports previous work conducted among Portuguese FFs that physical and cognitive stress symptoms increase during the working day, probably due to the experience of stress events [22]. These results suggest that this population may be experiencing potential cumulative stress effects along the days. Future longitudinal studies are required to confirm this assumption.
Finally, significantly negative and strong correlations were found between the stress symptoms reported by the FFs and some of the ECG-derived measures - AVNN, SDNN and pNN50 obtained during events (Table III).

TABLE III. Significant correlations between stress symptoms and ECGderived measures and correspondent values.

\begin{tabular}{|l|c|}
\hline Correlated variables & Spearman r value \\
\hline Stress symptoms ${ }^{2}$ vs AVNN & $-.829^{*}$ \\
\hline Stress symptoms ${ }^{1}$ vs SDNN & $-.943^{* *}$ \\
\hline Stress symptoms ${ }^{2}$ vs SDNN & $-.829^{*}$ \\
\hline Stress symptoms ${ }^{1}$ vs pNN50 & $-.943^{* *}$ \\
\hline Stress symptoms ${ }^{2}$ vs pNN50 & $-.829^{*}$ \\
\hline
\end{tabular}

${ }^{1}$ Mean of stress symptoms for the beginning of the shift; ${ }^{2}$ Mean of stress symptoms obtained at the end of the shift; *p $<.05 ; * * \mathrm{p}<.01$

These results showed that stress symptomatology (either physical or cognitive) at the beginning and at the end of shifts is related with a depressed HRV during stressful events. These results propose parasympathetic withdrawal, when experiencing stress symptoms [23]. Practical implications of these results could be, for example, a better organization time for rest and leisure or exercise program and/or change the cause instigating the stress symptoms.

\section{CONCLUSION}

In sum, there is evidence that stress is part of FFs routines, but they may not be truly aware of their stress levels, therefore, they are unlikely to ask for help. Further attention should be dedicated to these professional's occupational health by collecting not only information about what causes stress but also concerning its real impact on psychological and physical health. This study has limitations, particularly the reduced sample size and the fact that stress symptoms were correlated with fire events, without controlling the time of the event. However, this study reinforces the importance of research for the design of prevention and intervention plans and programs adapted to this hazardous population's real needs. Furthermore, the multi-dimensional and complex essence of stress among first responders demands that administrators, management authorities, and associated policy makers take a comprehensive approach for the management of stress in these occupations. Such approach will improve the well-being, security, and productivity not only of the individual workers but also the communities they serve. Future studies should be conducted with larger samples and with different professionals.

\section{ACKNOWLEDGMENT}

We would like to thank the Chief Command of the Portuguese firefighter department and the firefighters for accepted to participate in this study.

\section{REFERENCES} Cignitti, L., Baldari, C., "Psychophysiological Responses of Firefighters to Emergencies: 
A Review," The Open Sports Sciences Journal, vol. 7, pp. 8-15, 2014.

[2] S. A. Jahnke, W. S. Poston, C. K. Haddock, and B. Murphy, "Firefighting and mental health: Experiences of repeated exposure to trauma," (in eng), Work, vol. 53, no. 4, pp. 737-44, Feb 152016.

[3] Federal Emergency Management Agency, " Firefighter fatalities inthe United States in 2014," 2015

[4] F. Lazarus R.S., S., Stress, coping and adaptation. New York: Springer, 1984.

[5] W. B. Cannon, "Stresses and strains of homeostasis," American Journal of Medical Sciences, vol. 189, pp. 1-14, 1935.

[6] J. Taelman, S. Vandeput, E. Vlemincx, A. Spaepen, and S. Van Huffel, "Instantaneous changes in heart rate regulation due to mental load in simulated office work," (in eng), Eur J Appl Physiol, vol. 111, no. 7, pp. 1497-505, Jul 2011

[7] R. Castaldo, P. Melillo, U. Bracale, M. Caserta, M. Triassi, and L. Pecchia, "Acute mental stress assessment via short term HRV analysis in healthy adults: A systematic review with metaanalysis," Biomedical Signal Processing and Control, vol. 18, pp. 370-377, 2015/04/01/ 2015.

[8] X. Zhong et al., "Increased sympathetic and decreased parasympathetic cardiovascular modulation in normal humans with acute sleep deprivation," (in eng), J Appl Physiol (1985), vol. 98, no. 6, pp. 2024-32, Jun 2005.

[9] N. Pattyn et al., "Cardiac reactivity and preserved performance under stress: Two sides of the same coin?," International Journa of Psychophysiology, vol. 93, no. 1, pp. 30-37, 2014/07/01/2014.

[10] S. Rodrigues, M. Kaiseler, and C. Queirós, "Psychophysiological Assessment of Stress Under Ecological Settings," European Psychologist, vol. 20, no. 3, pp. 204-226, 2015/07/01 2015.

[11] J.P.S. Cunha, "pHealth and Wearable Technologies: a permanent challenge.," in Studies in Health Technology and Informatics, I. Press, Ed., 2012, pp. 185-195.

[12] J. P. S. Cunha, Cunha, B., Pereira A.S, Xavier W, Ferreira N, Meireles L.A. , "Vital Jacket: A wearable wireless vital signs monitor for patients' mobility in Cardiology and Sports," in 4th International ICST Conference on Pervasive Computing Technologies for Healthcare, , Munich 2010.

[13] S. Cohen, \& Williamson, G., "Perceived Stress in a Probability Sample of the United States.," in The Social Psychology of Health: Claremont Symposium on Applied Social Psychology, S. O. S. Spacapan, Ed. Newbury Park: Sage, 1988, pp. 31-67.

[14] F. X. Lesage, S. Berjot, and F. Deschamps, "Clinical stress assessment using a visual analogue scale," (in eng), Occup Med (Lond), vol. 62, no. 8, pp. 600-5, Dec 2012.

[15] T. Ritvanen, V. Louhevaara, P. Helin, S. Väisänen, and O. Hänninen, "Responses of the autonomic nervous system during periods of perceived high and low work stress in younger and older female teachers," Applied Ergonomics, vol. 37, no. 3, pp. 311-318, 2006/05/01/ 2006.

[16] "Heart rate variability: standards of measurement, physiological interpretation and clinical use. Task Force of the European Society of Cardiology and the North American Society of Pacing and Electrophysiology," (in eng), Circulation, vol. 93, no. 5, pp. 1043-65, Mar 11996.

[17] J. Pallant, SPSS Survival Manual: A Step by Step Guide to Data Analysis Using SPSS for Windows Version 15. Open University Press, 2007, p. 352.

[18] J. Pan and W. J. Tompkins, "A real-time QRS detection algorithm," (in eng), IEEE Trans Biomed Eng, vol. 32, no. 3, pp 230-6, Mar 1985.

[19] G. D. Clifford, F. Azuaje, and P. McSharry, Advanced Methods And Tools for ECG Data Analysis. Artech House, Inc., 2006.

[20] A. Voss, R. Schroeder, A. Heitmann, A. Peters, and S. Perz, "Short-term heart rate variability--influence of gender and age in healthy subjects," (in eng), PLoS One, vol. 10, no. 3, p. e0118308, 2015.

[21] S. Rodrigues et al., "Ambulatory Assessment of Psychophysiological Stress among Police Officers: a Proof-ofConcept Study," Occupational Health Science, journal article December 012017.

[22] P. Gomes, M. Kaiseler, C. Queiros, M. Oliveira, B. Lopes, and M Coimbra, "Vital analysis: annotating sensed physiological signals with the stress levels of first responders in action," (in eng), Conf Proc IEEE Eng Med Biol Soc, vol. 2012, pp. 6695-8, 2012.

[23] E. Hynynen, N. Konttinen, U. Kinnunen, H. Kyröläinen, and H. Rusko, "The incidence of stress symptoms and heart rate variability during sleep and orthostatic test," European Journal of Applied Physiology, vol. 111, no. 5, pp. 733-741, 2011/05/01 2011 